\title{
De la tradition juive à la critique sociale
}

La «Pensée du retour » chez Léon Chestov, Benjamin Fondane et Benny Lévy

Julia David

\section{OpenEdition}

1 Journals

Édition électronique

URL : http://journals.openedition.org/assr/8253

DOI : $10.4000 /$ assr.8253

ISSN : $1777-5825$

Éditeur

Éditions de l'EHESS

Édition imprimée

Date de publication : 1 septembre 2007

Pagination : 27-45

ISBN : 978-2-7132-2144-6

ISSN : 0335-5985

Référence électronique

Julia David, « De la tradition juive à la critique sociale », Archives de sciences sociales des religions [En ligne], 139 | juillet - sepembre 2007, mis en ligne le 19 novembre 2010, consulté le 02 mai 2019. URL http://journals.openedition.org/assr/8253; DOI : 10.4000/assr.8253 


\section{Julia David}

\section{De la tradition juive à la critique sociale La "Pensée du retour " chez Léon Chestov, Benjamin Fondane et Benny Lévy}

"Le retour au bercail jamais accompli, mais toujours imminent, où nous n'avons encore jamais été. [...] "Vous retournez où vous n'avez jamais été” ", George Steiner (2003)

C'est sous le signe de l'énigme que la " pensée du retour » fait son entrée dans l'histoire des idées, requérant d'emblée la souplesse du jugement, le va-et-vient de la nuance. Présente dans le corpus biblique, reformulée dans la littérature talmudique autour de l'expression de teshuva (du radical ShUV, revenir) l'idée de « retour » à la tradition juive s'affirme infiniment mobile : attachée au spirituel, elle tend en permanence vers le spéculatif; saturée de connotations morales et associée à l'idée de repentir, elle outrepasse pourtant les régions de l'éthique pour accueillir des exigences rituelles ou hilkhatiques; scellée au cœur de l'intime et des trajectoires individuelles, elle porte son message jusque sur la scène collective du politique et des aspirations nationales. Rappel en ombre chinoise d'un abandon du legs, d'une initiale trahison, elle infirme pourtant l'idée même d'exit hors de la tradition en réaffirmant l'indéfectibilité de l'Alliance ; fille de l'interruption et des caprices du désengagement, elle semble pourtant assurée de son antériorité silencieuse, vigile d'une présence toujours préalable à sa venue, préexistant mystérieusement, si l'on en croit la tradition, à la création même du monde ${ }^{1}$. Teshuva, terme fuyant s'il en est, riche de tant de significations que Franz Rosenzweig l'affirmait "irréductible(s) à toute expression différente du terme hébreu. » $(1935: 76)^{2}$. Pourquoi alors s'entêter, comme le fait Benny Lévy, à populariser l'expression de "pensée du retour " ? Pourquoi en déduire avec tant d'assurance que " c'est toujours par le retour qu'il y a du juif » (Lévy, 2003 : 29) ? N'y avaitil pas là, dans ces prudents refus de traduction, comme un avertissement sourd, une manière de poser la teshuva comme cet inapprivoisable se jouant des ghettos lexicaux? Benny Lévy n'est pourtant pas le premier à parler de "retour »;

1. T.B. Yoma 86b. Pirquey derabbi 'Eli’ezer, chap. 3. Midrash Ps. 90. Cité dans Gross (1998, p. 20).

2. Cité dans Gross (1998, p. 193). 
Emmanuel Lévinas, déjà, avait pu évoquer le "frisson du Retour » pour rendre compte du parcours de Rosenzweig, héros d'une légendaire teshuvah ce fameux Kippour 1913. Vocable têtu, récurrent, indépassable donc. "Retour " énigmatique si l'on considère pourtant le sort que la Bible réservait naguère à cette idée. Souvenons-nous de la femme de Loth, changée en colonne de sel pour avoir passé outre l'interdiction de regarder en arrière. Comme si, dans la tradition juive, tout retour était immédiatement suspect, comme si tout retour pétrifiait impitoyablement...

Ces ambiguïtés ne travaillent pas seulement le corps de la pensée juive ; bien au-delà des ruses du pilpoul ou des bizarreries de l'exégèse, c'est l'expérience même de redécouverte du judaïsme telle qu'elle s'impose dans l'épreuve de la modernité à partir de la fin du XVIII ${ }^{\mathrm{e}}$ siècle qui s'affirme paradoxale : opération de réinvestissement identitaire et culturel après les grandes fissurations de l'Émancipation, elle ne cesse pour autant d'illustrer le vertige d'une transmission problématique et d'un lien altéré ; derviche tourneur du rachat et de la reprise, volonté de rejouer l'inaugural, la «pensée du retour» ne cesse d'irriter la mémoire, d'inquiéter l'origine. Si la puissance de continuité dans ces expériences de ré-invention du judaïsme semble plus forte que dans d'autres traditions au regard de l'étroite imbrication, propre au fait juif, de l'identité et du religieux, notons que l'entreprise de reviviscence y est également plus fragile qu'ailleurs, soumise au feu nourri d'une histoire souvent tumultueuse. Des exigences particulières dictées par un statut minoritaire qui contraint les juifs à résister aux séductions de l'assimilation, aux ébranlements du siècle qui ont si souvent conduit le judaïsme au bord du gouffre, ces cheminements à rebours apparaissent singulièrement malaisés.

C'est sans aucun doute une double impossibilité - impossibilité de se dérober à l'histoire juive et impossibilité de la retrouver - qui conduit nombre d'intellectuels juifs sur le chemin de la critique de la modernité : la "réponse ${ }^{3}$ ne sera pas seulement religieuse, en effet; la "pensée du retour» se fait aussi critique sociale, indissociable d'une réflexion plus vaste sur la crise de la tradition et les impasses du progressisme historique. Dans la «pensée du retour » coexistent en effet deux mouvements distincts et complémentaires ; un élan de re-légitimation de la tradition juive, fût-ce sous des formes hétérodoxes, tout autant qu'une relecture critique de la pensée occidentale : ces penseurs sonnent non seulement le glas d'une certaine vision téléologique de l'histoire mais proposent aussi une remise en question de l'humanisme classique, dont ils dénoncent l'obsession subjectiviste et la tentation anthropocentriste. Ce double retour à l'œuvre chez la plupart des penseurs juifs d'Europe connaît pour autant des formes infiniment variées, fonction bien sûr des cultures nationales et des contextes historiques

3. C'est l'un des sens du mot teshuvah. 
- on ne « retourne » pas au judaïsme après la Shoah comme pendant l'entre-deuxguerres - mais propres aussi à chaque biographie, dans l'audace du tâtonnement et des secrets partagés, souvent bien au-delà du cercle de la tradition. Pensons au judaïsme weimarien, à ce "paysage juif commun" selon l'expression de Rosenzweig, qui fut en réalité cette Babel du retour, chevauchement miraculeux de langues et d'espérances toujours plurielles; retour nimbé de mystères chez Rosenzweig, happé, selon la légende, par quelque inexplicable réveil dans la « nuit de Leipzig »; retour bien plus mélancolique chez Scholem, tournant résolument le dos au "marché de dupes » de l'assimilation; retour révolutionnaire chez Benjamin aimantant l'utopie marxiste au messianisme de la tradition et considérant avec scepticisme le rêve sioniste de son ami berlinois ; retour ascétique chez Leo Strauss, choisissant de réinvestir, loin des rhétoriques romantiques, l'héritage du rationalisme classique et des Lumières médiévales, stratège et funambule explorant les tensions jusqu'au vertige... Resterait bien sûr à évoquer les brisures propres à chaque parcours, quand le "retour» finit par crever sa gangue d'illusion pour s'inventer autrement; retour pour Scholem, De Berlin à Jérusalem, mais voyage symbolique aussi, au soir de sa vie, de Jérusalem à Berlin (Biale, 2001 : 240), comme si la rupture avec l'esprit diasporique s'avérait finalement tout aussi utopique que ne l'avait été l'ancien idéal de symbiose... Parler de "pensée du retour ", mais à la seule condition alors de l'envisager comme un vaste champ de bataille où se succèderaient ordres et contre-ordres, assauts désordonnés et tir croisés, dans l'amoncellement anarchique des deuils et des renaissances.

En France aussi, et peut-être plus encore dans un pays où la Révolution aura radicalisé les espérances tout autant que la crise de conscience, les retours se suivent et ne se ressemblent pas; quoi de commun à vrai dire entre le retour tardif de Julien Benda, pur produit de l'assimilation épris de classicisme grec, louchant au soir de sa vie vers les prophètes juifs, en dilettante du judaïsme, et le retour fiévreux de son contemporain Fondane, remontant patiemment le cours de l'histoire, des avant-gardes européennes au monde traditionnel de ces lointains tîrguri de Moldavie où chantait encore le yiddish (Iancu, 1978) ? Quelle affinité possible entre le retour au judaïsme d'Edmond Fleg, survenant au lendemain de l'Affaire Dreyfus comme un pied de nez aux affronts de la rhétorique maurassienne, s'affirmant comme une réponse universaliste à la violence des ethnies, et le retour au judaïsme de cet exilé russe que fut Léon Chestov, brandi, au lendemain de l'universalisme terroriste de la révolution bolchévique, comme le front de résistance d'une petite tribu conservatrice ? Surtout, quoi de commun entre l'entreprise de renaissance de la philosophie existentielle juive de l'entredeux-guerres empruntant encore ses catégories aux "nations ", et le paysage spectral de l'après-Shoah ? Comment postuler, même, une quelconque proximité entre le retour d'Emmanuel Lévinas et le "tournement» de Benny Lévy ? À l'aventure lévinassienne impulsant un retour perpétuel, retour invisible et sans 
âge, baigné comme de toute éternité dans les eaux de la tradition, répond le retour explosif de Benny Lévy, assumant tous les rôles dans ce conte oriental où l'imposture précède et dissimule la promesse...

Déroutant panorama s'il en est, où toujours les « juifs du retour » ridiculisent la synthèse, éreintent les fictions théoriques. On pourrait pourtant, sous ce voile de confusion, distinguer à grands traits trois figures de retour; retour de ces juifs déjudaïsés condamnés à tout reprendre, à tirer de quelques oripeaux de grenier, misérable folklore, la substance vitale de leur résurrection ; retour en forme de spirale pour ces juifs demeurés dès l'enfance à l'horizon de la tradition juive et qui font du " retour " plus qu'un itinéraire personnel dont on chercherait en vain les drames et les tremblements, une métaphore du judaïsme même, s'enroulant au plus près de son axe ; retour enfin de ceux qui, nés dans un univers encore gorgé des préceptes de la tradition, ont préféré s'éloigner pour s'engager résolument dans les convulsions de l'âge moderne, puis, finalement dégrisés, redécouvrent la source, mais déniaisés par l'expérience du siècle. Ce sont ces derniers trajets qui ont ici retenu notre attention : biographies denses qui ramassent en l'espace d'une seule vie cette valse à trois temps qui court le plus souvent sur plusieurs générations, tradition - rupture - tradition. Avec Léon Chestov, Benjamin Fondane et Benny Lévy, le retour est en effet à échelle humaine; ils ne sont pleinement les acteurs du retour que parce qu'ils ont été eux-mêmes les protagonistes du divorce. Alors que les juifs allemands de l'entre-deux-guerres entendaient conjurer un renoncement dont ils avaient hérité bien malgré eux, ces juifs là, issus d'une Europe orientale ou d'un bassin méditerranéen encore marqués par le poids de la tradition, sont tout à la fois les enfants rebelles qui quittent la demeure et les enfants qui s'acquittent de la dette, ceux qui trahissent et ceux qui accomplissent. Avec eux, en effet, il est un temps pour la révolution et un autre pour les textes juifs, un temps pour les avant-gardes et un temps pour la critique religieuse du modernisme, un temps pour les «lendemains qui chantent " et un temps pour l'idée de négativité retrouvée dans l'éclat sombre des lettres carrées. Au-delà des diversités propres à chacun de ces parcours, c'est cette expérience infiniment moderne de la volte-face, ce choix, en connaissance de cause, d'une tradition qu'ils avaient d'abord, non seulement désertée mais répudiée, qui donnent à leur critique de la modernité une profondeur si singulière. Acrobates des deux rives, ennemis de l'homogène, n'auront-ils pas finalement « dépayser » l'héritage pour mieux troubler le jugement des Modernes?

\section{" Juifs du retour " et crise de la modernité}

Déjà au XIX ${ }^{\mathrm{e}}$ s'amorce un retour à la tradition juive qui est aussi une brèche dans la culture politique moderne. En effet, ce siècle que l'on a souvent présenté comme celui du tarissement et des conversions au christianisme - pensons à la propre descendance de Mendelssohn - fut aussi celui du « retour » à la tradition 
juive d'Hermann Cohen, redéployant, en une refonte critique du kantisme, la morale et les catégories abstraites des Lumières du côté d'une hétéronomie positive, celle dont l'héritage hébraïque tissait pour lui le filet protecteur. Retour aussi, soulignons-le, jusque chez les convertis juifs au christianisme, où le murmure de la source continuait d'intriguer et de contrarier la doxa assimilatrice. Pensons en effet à Heinrich Heine, enfant terrible des Lumières s'il en est, apôtre du saintsimonisme et de l'émancipation du genre humain, " exemple anticipé de l'homme moderne " selon la formule d'Heinrich Mann, et qui n'aura pourtant de cesse de désavouer cette "vaste et désagréable modernité » (Höhn, 1994 : 98). Acquises aux valeurs de l'humanisme progressiste, emblématiques par excellence de ce cosmopolitisme dont les Lumières faisaient une promesse de fraternité, bénéficiaires reconnaissantes de l'Émancipation, frottées au projet libéral de la Wissenschaft des Judentums - rappelons que ce fut le cas de Cohen mais aussi de Heine - les élites juives, fussent-elles converties comme le poète des mélodies hébraïques ou profondément rétives aux pesanteurs de l'orthodoxie, avaient, depuis bien longtemps déjà, cherché auprès de la tradition juive de quoi contrer les effets négatifs du processus de rationalisation occidentale. Le scepticisme était donc ancien ; on pourrait dire qu'il fut d'une certaine façon consubstantiel à l'entrée même des juifs dans la modernité ; être moderne, n'était-ce pas fondamentalement, après tout, se montrer apte à la critique ?

Mais avec le fantastique détonateur de la Grande Guerre, l'urgence d'une reformulation du projet moderne changeait encore de nature. L'évènement qui avait mobilisé tant d'énergie dans la défense des vertus nationales, de la défense de la « germanité » chez Cohen ou Buber au patriotisme vivace des juifs français, que ni l'Affaire Dreyfus ni les campagnes antisémites des années 1880 n'avaient réellement ébranlé, marquait symboliquement dans les esprits la décomposition de cette ère libérale déjà mise à mal par l'essor des mouvements révolutionnaires, signait le discrédit de cet " embourgeoisement » juif (Verbürgerlichung) qui avait constitué, par-delà la dissemblance des modèles français et germanique, le nerf commun de l'assimilation. L'entre-deux-guerres s'annonçait pour la plupart des intellectuels juifs sous le signe incontestable du dégrisement. Que le caporal Rosenzweig adresse du front de Macédoine ses lettres au Dr Rosenzweig de Cassel suffirait à dire la brisure du Sujet : L'Étoile de la Rédemption, expulsant sa plainte sous l'impérieuse dictée du " regard du mourant ${ }^{4}$, se présente alors comme une formidable dénonciation de l'idéalisme allemand. Pensons également

4. On peut lire, sur cette question, la préface de Stéphane Moses à L’Étoile de la Rédemption, qui met bien en lumière le traumatisme que représenta pour Rosenzweig la guerre de 1914-18 et la désaffection qui suivit vis-à-vis des idéaux des Lumières : « [...] devant le spectacle du carnage insensé auquel se livrent les nations européennes - celles-là mêmes qui avaient inventé l'idéal philosophique d'un monde régi par le Logos - il n'est plus possible d'affirmer que le réel est rationnel, ou qu'à la lumière de la Raison le chaos originel se transforme nécessairement en un cosmos intelligible. » in préface de Stéphane Moses à L'Étoile de la Rédemption, Paris, Seuil, 1982 et 2003, p. 11. 
à Gershom Scholem, évoquant dès 1916, la " mise au tombeau " ${ }^{5}$ des héritages européens. Pour les élites juives, une certaine euphorie, celle de l'assimilation, du progrès, et plus globalement de la modernité, connaissait là sa première grande défaite.

Issus d'Europe orientale, Léon Chestov et Benjamin Fondane n'échappent pas davantage au traumatisme. Pour les deux hommes, la guerre est un temps de basculement : Chestov y perd son propre fils; Fondane se voit extirpé, selon ses termes, de son "sommeil idéaliste ». Avec la vision d'une "terre toute ventre sur le billard des hôpitaux » et la révélation d'un "vieux monde écroulé » dont la décomposition venait manifester sans fard «tous les trucages» (Fondane, 1999 : 77) du rationalisme moderne, les « miracles » de la science apparaissaient désormais proportionnels à la progression de l'incertitude; le rêve de maîtrise sombrait alors même que la raison scientifique semblait triompher : la célébration de la commune humanité de Nathan, Saladin et le Templier paraissait bien lointaine à l'heure où la raison universelle kantienne se voyait balayée sous le déchaînement des intérêts particuliers.

Pourtant, impitoyables à l'endroit de la raison kantienne, moqueurs de la confiance naïve dans les pouvoirs illimités de la raison objective, Chestov et Fondane se refusaient encore à répudier les dernières promesses des Lumières ; les mauvais sorts de la connaissance pouvaient encore se voir conjurés. Avec Benny Lévy, la critique se fait bien plus radicale. Enfant de l'après-Shoah, sa critique de la modernité perd définitivement foi dans les vertus critiques ellesmêmes. Si Lévy s'agace du topos faisant d'Auschwitz le lieu de la "mort de Dieu ", nul doute pour lui que la philosophie occidentale s'y consume entièrement. Brutal arrêt sur image : " fin de la philosophie, écrit-il ; la philosophie se suspendant fait lieu à la pensée du Retour. " Si la Pensée du retour n'entame pas le messianisme de la tradition, et affirme, plus que jamais, la chimie explosive d'un possible "virement de la malédiction en exultation ", c'est pour mieux l'opposer pied à pied au scandale des théodicées rationnelles de l'Occident. Loin d'interroger le principe espérance de la tradition religieuse, c'est la raison moderne et ses équations providentialistes, l'esprit de la dialectique hégélienne, que le grand fracas aurait pulvérisés.

À l'expérience des deux guerres mondiales s'ajoute l'effondrement des grandes utopies révolutionnaires, des grandes «mystiques du peuple ». Brûlés par cette révolution bolchévique dont Chestov devait dénoncer l'utopie dévastatrice dès 1919 dans Qu'est-ce que le bolchévisme? Fondane et Chestov s'éloignent dès l'entre-deux-guerres, de l'optimisme historique pour lui opposer désormais la chute et le péché biblique. Quant à la génération de Benny Lévy, elle assume l'épilogue de ce désenchantement, projetée du repoussoir totalitaire vers les radicalités des années 1960 et 1970, avant d'en dénoncer à nouveau l'illusion.

5. Cité dans Moses (1992, p. 22). 
Ainsi, le retour est d'abord l'aventure d'une déroute ; interprété comme le dernier acte de l'épuisement des idéologies modernes, il entend prendre la mesure des impasses du «tout-politique ». Mais le retour n'est pas seulement l'envers du décor philosophique et politique de l'Occident, il a son aliment propre, il n'est d'abord possible que parce qu'une généalogie silencieuse en porte l'extrême tension.

\section{Retour aux fondations ou re-fondation?}

\section{Fondations}

Poids d'abord de la tradition. Au commencement était le rite. Pour Chestov, pour Fondane, pour Benny Lévy, il y a une enveloppe matricielle, une berceuse, un chant lancinant, celui d'une enfance juive. Pas d'arbre de Noël comme chez Scholem, pas de «bourse vide » comme chez Kafka, contemplant son infortune, « tout nu, au milieu de lambeaux et de fragments » 1988 : 261). Pas de judaïsme assimilé délivrant quelques maximes évanescentes, nulle "tradition tombée malade " ${ }^{6}$ selon l'expression cruelle de Walter Benjamin, mais l'ossature d'un judaïsme compact, l'expérience première d'un judaïsme allant de soi. Né à Kiev dans la seconde moitié du XIX ${ }^{\mathrm{e}}$ siècle, dans le quartier de Podol, Léon Chestov grandit dans une famille juive encore fortement marquée par les prestiges de la tradition. On reçoit dans la demeure familiale Sholem Aleïkhem, on fréquente la synagogue, on célèbre les grandes fêtes juives. Son père ne fut pas seulement cet apikoïrès (libre penseur) moquant les fanatiques, trublion sympathique choisissant, au grand dam des rabbins, les bancs de la synagogue pour raconter ses histoires drôles, ce commerçant appartenant à la première ghilde fut aussi un grand connaisseur des textes hébraïques, recrutant même pour ses enfants un professeur d'hébreu. " [...] lorsque le jour d'une fête, confiait-il, on porte dans la synagogue les saints rouleaux de la Torah, je les baise. »(Baranoff-Chestov, 1991 : 15-16). Certes, les parents n'observent déjà plus shabbat, mais une cousine se charge de dresser la nappe blanche et d'allumer les bougies, devant l'enfant attentif. Quelques gestes donc, étranges et familiers ; aura de mystère, petit balluchon légué pour le restant d'une vie. Avant que d'être philosophie ou catéchisme éthique, le judaïsme fût ici un judaïsme vécu; le philosophe existentiel devait s'en souvenir. Même Stimmung religieuse chez son disciple et ami Benjamin Fondane : né à Jassy, une petite bourgade juive de Roumanie connue pour son théâtre yiddish, il passe ses vacances à Hertza, un schtetl juif typique, d'où étaient originaires ses grands-parents paternels. Milieu lettré - son grand-père maternel, venu de Galicie, poète de langue hébraïque et fondateur des premières écoles juives de Moldavie devait lui léguer fort symboliquement sa bibliothèque - à l'écoute de la culture européenne et, pour autant, intensément juif dans ses

6. Lettre de Benjamin à Scholem, 12 juin 1938, in Walter Benjamin, 1979, vol. II, p. 250. 
aspects les plus concrets. Fondane de décrire en effet les chants hébreux du seder de Pessah, le ronron musical des prières ou, encore, les tombes du cimetière juif de son enfance. Judaïsme éclairé où résonne la poésie yiddish de Jacob Groper, et maintenant pourtant sans complexes les rênes de la tradition dans ses exigences les plus formelles. En considérant l'enfance de Fondane comme celle de Chestov, très loin de celles décrites par la plupart des grandes figures du judaïsme allemand, on pense inévitablement à cette formule de Rosenzweig : alors que "le Westjude regarde toujours, avant d'oser s'asseoir, si une chaise est là ", le Ostjude " a sa chaise derrière lui et s'assoit dessus sans regarder autour [...] " ${ }^{7}$. C'est cette assise-là, ces fondations cimentées dans les profondeurs qui autorisent la possibilité ultérieure du retour. Si Benny Lévy n'est pas un Ostjude, il partage néanmoins cette sollicitation première, ce judaïsme sans crise identitaire, alors posé dans son évidence, dans sa tranquille persévérance. Comme Benjamin Fondane, comme Léon Chestov, Benny Lévy aura bu à l'eau du puits. Judaïsme sans intellectualité, confessait-il pourtant, mais traversé par l'histoire en chacun de ses plis et replis. Évoquant le Caire de son enfance, Lévy affirme ainsi, "Je viens de là, de cette ville par laquelle est passée l'Ari ha-kadoch, l'Ari zal. " ${ }^{8}$ Et il ajoutait, "Je dois à mes grands-parents et à ma mère, essentiellement, d'avoir eu cette possibilité de retour dans les grands paysages de mon enfance. " ${ }^{9}$

Ainsi, le retour ne ferait qu'officialiser une présence, confirmer une précédence plus coriace que l'offense ; le rappel à l'ordre serait tout entier embusqué dans ces balbutiements là, riche de cette seule genèse. C'est l'idée, chère à Benny Lévy, de dévoilement (gilouï en hébreu) : le viatique n'aurait pas été perdu, il n'aurait pas été corrompu, mais voilé. "J'étais un juif oublieux de moi-même " (Lévy, 2004 : 53) affirme l'ancien dirigeant maoïste. Parce qu'une allégeance antérieure aurait été scellée et continuerait en secret de faire entendre sa pulsation, le juif amnésique n'est pas un juif débouté mais un juif en sursis; il est celui qui « dit la vérité sans le savoir»(Ibid. : 110), celui pour lequel la petite «loupiote de la Guéoula» déjà fait signe, dans l'espace même de la confusion. Ruse du psychisme disculpant l'infidèle, fantastique machine à re-virginiser les consciences ? Constatons seulement qu'il y a là une parole très différente des autres récits traditionnels de conversion. Nulle transfiguration spectaculaire, nulle illumination sur le chemin de Damas, pas de «temporalité éclair » (Badet, 2002 : 181) ou de nuit pascalienne. Pas de ravissement claudélien derrière un pilier de cathédrale. Nulle vision, comme chez Max Jacob, « visité » dans sa chambre de la rue Ravignan. La «pensée du retour » n'induit aucune métamorphose, aucun de ces séquençages tranchés et décisifs qui firent le bonheur de l'iconographie occidentale. Car elle se veut d'abord et avant tout, réaffirmation d'une origine irrécusable. "Groper,

7. Cité dans Bouretz (2003, p. 203).

8. Rabbénou Itzhak ben Chlomo Louria Askénazi (1534-1572). In Cahiers d'études lévinassiennes, Hors série, 2005, p. 9.

9. Ibid. p. 11. 
écrit Fondane, m'a pris par la main et m'a mené vers le portrait de mon aïeul : Benjamin Schwarzfeld. J'ai compris que là était la tradition. Je suis revenu aux origines et à la Bible. » (Fondane, 1919). Benny Lévy ne dit pas autre chose, qui entend revenir "sans problème, à la "foi de nos pères” " (Lévy, 2003 : 17). Dès lors, il n'y a pas conversion mais conversation: tête-à-tête avec les Anciens, ré-appropriation d'une filiation qui coule de source. Les " Juifs du retour » rapatrient ce qui jamais ne fut quitté, cet indéracinable point d'intimité décourageant les plus apostats. "J'ai tout essayé pour fuir, me dérober ", dira Fondane (1996), marquant bien l'impossibilité d'échapper à la question. Sous les écarts et les intermittences, la "pensée du retour » réaffirme l'unicité du trajet; la permanence est son royaume.

\section{Refondation}

Manifestation d'une indépassable continuité qui la rendrait presque superflue, paradoxal déni de bifurcation, sinuosité détrompée par l'aplomb de ce qui fut donné au commencement, la " pensée du retour » est pourtant tout autant le lieu du vertige moderne : pas de " happy end » en effet, pas de circularité miraculeuse, pas de purge vertueuse. La "pensée du retour» n'a rien d'un simple retour à l'origine. "Rien ne me manque ${ }^{10}$, lançait comme une provocation Benny Lévy au terme du parcours, comme pour asseoir une plénitude, confirmer la victoire, authentifier la reconquête. Pourtant, tout n'est pas si simple, pas de boucle bouclée en vérité, pas de retour au bercail pour ces "Juifs du retour ", mais une double obsession : celle du legs comme un « reste » opiniâtre, impossible à solder; celle d'une tradition aux contours insaisissables, chahuteuse, impossible à amarrer. Encore trop présente pour être oubliée, mais trop frêle pour être capturée, suffisamment vivace pour intriguer et faire entendre sa plainte, insuffisamment dense pour consoler. Cette ambivalence fondamentale se vérifie ici tout particulièrement : on ne le rappellera jamais assez, Chestov, Fondane et Lévy ne sont pas des juifs occidentaux mais des juifs de l'entre-deux, un pied dans un univers encore marqué par le poids de la tradition, un pied dans la modernité de l'exil. Trop proches de la tradition pour l'idéaliser dans ses aspects " primordiaux », trop curieux d'un Occident dont ils n'ont fait qu'entrevoir les promesses pour succomber au charme des traditions perdues et du Volkstum communautaire, ils demeurent également trop marginaux après leur exil pour reproduire les fragiles équilibres des juifs occidentaux.

\section{Contre le retour « romantique » à la tradition}

L'intérêt pour le mythe biblique, un mythe que les enchantements de la rationalité moderne auraient édulcoré, les rapproche incontestablement de

10. Psaumes, XXIII. Cité dans Lévy (2002, p. 8). Repris dans Lévy, Finkielkraut (2006, p. 165). 
Scholem et de Buber, tant ces derniers plaçaient la réhabilitation des éléments mythiques du monothéisme au centre de leur réinvention de la tradition.

Leur entreprise évolue pourtant loin des accents nostalgiques de ce néoromantisme juif. Souvenons-nous en effet du retour des penseurs juifs allemands. Nostalgie d'un âge héroïque chez Martin Buber revisitant la guerre des Maccabées et la " destinée exceptionnelle du héros » (Buber, 1986 : 87), appel à une " religion romantique ${ }^{11}$ pour Scholem (1999: 265). Leur intuition d'une crise de l'intelligence et leur représentation d'un crépuscule de la culture s'inscrivent alors dans une grille de lecture nietzschéenne: pensons à Buber empruntant la notion de neue Gemeinschaft au groupe nietzschéen dirigé par les frères Hardt au tournant du siècle, opposant à la " solitude massifiée » du monde moderne, l'immémoriale communauté de l'Urmensch... Pour cette génération, il s'agit alors non seulement de faire face à la disgrâce du rationalisme occidental, de résister aux "normes obtuses des Lumières " ${ }^{12}$, de contrarier la lente agonie matérialiste des sociétés, mais de repenser aussi la vocation du judaïsme comme fondamentalement contraire à la culture « bourgeoise », en l'associant à la vitalité communautaire et "dionysiaque » du judaïsme oriental. Pour Scholem à la pension Struck, pour Buber se remémorant la Galicie des grands-parents, la culture des Ostjuden apparaît alors comme la source vive du judaïsme, redessine pour eux les contours d'un monde enchanté. Que cette idéalisation résonne en réalité comme une figure du remord, toujours est-il que cette nostalgie même illustrait, mieux que toute autre posture sans doute, leur enracinement irréversible dans la modernité occidentale. Il n'est qu'à penser au thème du retour à la nature qui accompagnait certains intellectuels juifs dans leur redécouverte du hassidisme et empruntait bien davantage à l'idéologie des Wandervögel qu'à la tradition hébraïque.

Le tableau est ainsi des plus paradoxal : alors que ces juifs allemands recherchaient auprès du judaïsme d'Europe centrale le sceau d'un judaïsme plus originel, ni Chestov ni Fondane, pourtant issus de l'univers des Ostjuden, ne succombent à la fascination de l'authentique. Pas d'ivresse des commencements chez eux, pas « d'appel nietzschéen à une cure de jouvence destinée à enrayer la décadence européenne » (Mattenklott, 1991 : 99), pas d'emballement en faveur d'un corps national à régénérer - leur retour, soulignons-le, ne s'engage d'ailleurs pas dans l'orbite du sionisme. Chez Benny Lévy, non plus, nulle recherche d'un analgésique de l'affiliation contre les maux désintégrateurs du monde moderne, nul sésame communautaire. Leur combat se mène bien loin de ces aspirations à la re-cimentation forcenée du social, bien loin des rêveries romantiques autour d'un âge édénique passé. S'ils refusent pour leur part d'évoquer la tradition en termes magiques, c'est qu'ils en auront été des acteurs effectifs : ils sont en effet les témoins directs - et donc fondamentalement ambivalents - des derniers feux

11. Cité dans "Mysticisme et société : un paradoxe créateur ».

12. Lettre de Scholem adressée à Zalman Schocken intitulée "Ein offenes Wort über die wahren Absichten meines Kabbalastudiums », 1937. Cité dans Biale (2001, p. 57). 
d'un univers traditionnel. Si la tradition n'est jamais présentée comme ce havre disparu, cet asile de vérité tragiquement éclipsé, c'est tout simplement qu'ils viennent à grand peine de lui échapper. Suffisamment émancipées pour donner le goût naissant de l'assimilation malgré les limites qu'imposent les sociétés dans lesquelles elles vivent, les familles de Chestov, Fondane et Lévy sont encore suffisamment soumises au joug de la tradition, suffisamment rivées aux instances communautaires qui les protègent d'un monde encore hostile, pour finir paradoxalement d'en éloigner leurs enfants. Mère nourricière, la tradition est tout autant ressentie comme un archaïsme aliénant, une mare stagnante, réceptacle de vieilles superstitions rendues surannées par les percées de l'occidentalisation et l'appel du large de l'universalisme moderne.

À la croisée des mondes, Chestov, Fondane et Lévy inaugurent ainsi leur périple sous le signe de la sédition, en rébellion contre une tradition jugée asphyxiante, mal accordée aux exigences du siècle. Les ressorts endogamiques seront ainsi impitoyablement brisés : mariage mixte pour Chestov sous le sceau du secret, changements de nom - pour Chestov comme pour Benny Lévy - qui ne doivent rien au hasard et disent bien le malaise dans la filiation; autant de dissidences, explicites ou voilées, pour rompre le pacte des obligations traditionnelles. Si l'on compare ces trajets au néo-romantisme des penseurs juifs allemand, le chassé-croisé est tout à fait net : alors que ces derniers soubaitaient rompre avec l'assimilation, Chestov, Fondane et Lévy commencent par déserter la tradition. Mais happés par la musique des Lumières nées à l'Ouest, délaissant les formes de vie juive traditionnelle, leur adhésion à l'héritage des sociétés occidentales demeure en réalité bien fragile. On songe à la métaphore du glacier reprise par Isaiah Berlin pour évoquer ces juifs qui n'ont réussi ni «à rester gelés ni à s'évaporer " : s'ils ont sans nul doute entamé leur dégel, s'ils ont défait depuis longtemps le nœud du rite, les promesses universalistes qui ont séduit les Juifs d'Occident continuent pourtant de sonner creux à leurs oreilles. Leur statut d'extraterritorialité vis-à-vis de l'histoire occidentale les aura éloignés tout autant des certitudes du rationalisme moderne que de la riposte irrationaliste qui fut son symétrique tout au long du siècle : trop étrangers encore à l'Occident pour s'abandonner à la Raison moderne, en devenir les dépositaires enthousiastes, ils furent aussi trop marginaux pour s'adonner à la nostalgie anti-moderne des juifs assimilés.

Surtout, alors que le retour aux sources des juifs allemands associait le mythe à des forces révolutionnaires, voire apocalyptiques, toujours, en tout cas, irrationnelles, loin de la pure intellectualité et des contraintes légalistes, eux n'eurent pas l'oreille suffisamment musicale pour succomber aux sirènes du mysticisme. Pas ici, en vérité, de prose bubérienne enflammée appelant à "réaliser Dieu ». Leur ferveur se déclina toujours sous les auspices d'une "autre " raison. Si la pensée de Léon Chestov comme celle de Fondane s'est développée en affinité avec les tâtonnements métaphysiques de l'entre-deux-guerres, attentive à la mystique 
romantique de Jules de Gaultier destituant les « idoles du ciel logique » (Fondane, 2001 : 203) ou fraternelle à l'endroit du mysticisme de Francis Jammes, ni Chestov ni Fondane ne s'engagent à l'horizon de la croyance. Drôles de mystiques qui n'ont jamais connu l'extase mais se passionnent bien plutôt pour les formes de cette autre rationalité dont ils empruntent la forme aux analyses de Lévy-Bruhl. Pour Benny Lévy également, c'est bel et bien "adossé au Logos » que le mythe «donne à penser énigmatiquement » (Lévy, 2005a : 103). Désireux de s'inscrire dans le sillon du Gaon de Vilna, c'est-à-dire dans la grande tradition rationaliste lituanienne, Lévy affirme ainsi, « je n'ai pas eu de glaive dans les reins, aucune vision, aucune hallucination... Je n'ai pas “CRU” - c'est un peu obscène cette croyance. Rien de tel : on travaille et on lern - on étudie " (Lévy, 2005b : 22). Et de poursuivre, mi-amusé mi-frondeur, "Je pense que j'étais encore athée lorsque je suis entré à la yeshiva de Strasbourg - c'est vous dire!». Loin des tonnants oracles de la Providence, le retour est ici retour aux palpitations du texte, à la puissance d'une langue - pensons à Benny Lévy qui apprend l'hébreu mais aussi l'araméen -, orthopraxie de ce na'assé vénichma réactualisant les gestes du souvenir.

\section{La dette inavouable ? Des héritiers de la modernité}

Cette prééminence des aspects « rationalistes » dans leur ré-évaluation de la tradition juive n'est pas le fait du hasard. Elle consonne avec la trajectoire moderne qui est la leur. En effet, la crise de la transmission n'est pas seulement un indisponible, une perte, une béance, comme on l'a souvent décrite, elle est aussi l'aventure d'un surplus, d'un excédent, cet acquis que la traversée du moderne a déposé, bagage tout aussi stimulant qu'encombrant pour ces " Juifs du retour ». Envers et contre tout, Chestov, Fondane et Lévy sont des archétypes de cette modernité qu'ils dénoncent. D’abord parce qu'ils illustrent cette possibilité du choix dans la construction de l'identité, possibilité qui constitue l'une des figures par excellence de la modernité ${ }^{13}$. Mais surtout, parce que l'on ne s'exclut pas de la modernité par simple fiat.

La modernité, de fait, les traverse et les constitue. Révolutionnaire dans sa jeunesse, proche de Narodnaïa Volia, Léon Chestov consacre alors une thèse à la condition ouvrière qui sera refusée en raison de ses accents subversifs. Séduit par le marxisme, il ne s'en détache qu'avec l'apparition d'un socialisme «scientifique ». Benjamin Fondane partage un temps les options tumultueuses des avant-gardes. "Tuons nos morts! » lançaient alors ses amis de Contimporanul ${ }^{14}$. Benny Lévy s’enflamme pour la thématique révolutionnaire sous le parrainage d'Althusser à l'École normale supérieure, fonde la Gauche prolétarienne avant de participer à la création du journal Libération en 1973. Trois baptêmes du feu marquant le passage à la radicalité moderne, trois expériences surtout qui

13. Sur cette question, voir Hervieu-Léger (1999).

14. Ion Vinea Contimporanul, "Manifeste activiste pour la jeunesse », 46, mai 1924. Cité dans Carassou, Releanou (1999, p. 11). 
ne s'abolissent pas dans la rupture, comme par magie, mais continuent bien plutôt de peser de tout leur poids sur les formes mêmes du retour. Le témoignage de Benny Lévy évoquant ses années de "perdition " est ici révélateur : "Si on laisse de côté le flash-back et que l'on en vient à une proposition rationnelle sur ces résonances que j'entendais alors, je dirais : quelque part cette radicalité a dû, intellectuellement - je ne parle pas du fond de mon être, mais intellectuellement, dans les formations intellectuelles - m'aider à m'approcher de la figure vraie du casseur d'idoles, j’ai nommé Abraham»(Lévy, Finkielkraut, 2006 : 114). Au demeurant, s'il retourne aux textes juifs, n'est-ce pas d'abord sous la guidée de Sartre, ou tout du moins dans le sillon de son « désir »? N'était-ce pas lui déjà, le maître du déclic, autorisant la réhabilitation du patronyme, balayant Pierre Victor comme un remord ? On a en effet beaucoup évoqué l'influence du fougueux et jeune Juif sur le dernier Sartre, resterait à saisir la pensée Sartre à l'œuvre chez le brûlant talmid 'hakham. Dans son livre sur La Pensée du retour chez Emmanuel Lévinas, Benny Lévy ne rend-il pas hommage, grâce à une formule lévinassienne, et dès la première page, au philosophe de l'existentialisme ? Sartre, qui joua "un grand rôle dans notre audace à revenir à de vieux discours interrompus depuis longtemps »... Sartre en homme-orchestre du Retour, on voit bien que l'aventure n'a rien, dès lors, de très... orthodoxe. Souvenons-nous encore de ces compagnons plus passagers mais tout aussi improbables, et dont on peut penser qu'à défaut d'influence décisive, ils initièrent tout du moins Lévy à un certain "climat ", autorisèrent son envol: pensons à Maurice Clavel, le catholique "born again ", auquel Lévy emprunte la formule de "journalisme transcendantal », et plus largement, la thématique du retour au désert ; Clavel ou l'énigme d'une résistance possible à la langue de bois marxiste. Pensons à cette expérience moderne que fut la grève-occupation de Lip, conjugaison baroque de la question sociale et de la question religieuse, où l'on vit le leader déjà déçu de la Gauche prolétarienne dialoguer avec l'ancien dominicain Jean Raguenès, et pressentir peut-être d'autres issues... Pensons à cet insolite Cercle socratique, dont Lévy est alors l'éphémère directeur, et qui sera tout en même temps, la marmite en ébullition d'une modernité de gauche impatiente de revoir ses catégories, et, déjà, l'attente d'une re-fondation faisant appel aux Anciens... Qu'est-ce à dire ? Qu'il y eut un Benny Lévy chrétien ? Puis un Benny Lévy grec ? Rien de tel bien sûr, mais sans doute un Benny Lévy plus fils de son temps qu'on ne le pense dans son mouvement même de retour; avide, comme beaucoup de ses contemporains d'alors, de "convertir » la formidable énergie révolutionnaire, de recycler, en le désorientant, son credo d'espérance. La " pensée du retour » s'inscrit pleinement dans l'histoire des idées modernes, tout à la fois indice de la crise de la modernité occidentale et tentative - heureuse ou malheureuse pour y résister.

Mais il y a plus encore. Car non seulement leur retour s'invente grâce à ces petits cailloux semés par la modernité elle-même, mais l'expérience moderne 
est également réintégrée dans le corps même de la pensée nouvelle. Évoquons le cas, en apparence le plus extrême, incarné par Benny Lévy : de Mao à Moïse ${ }^{15}$ titraient les amateurs d'insolite, oubliant, sous Mao, un premier Moïse, insistant, dur au mal ; mais sous le dernier Moïse, le Moïse de la réconciliation, c'est cette fois le cœur intellectuel des luttes modernes qui bat encore. Ne continue-t-il pas, kippa et phylactères "ressuscités ", à batailler avec Husserl ? Ne garde-t-il pas des forces pour affronter Spinoza ? Étrange orthodoxe qui n'en finit pas de chercher querelle à la philosophie, qui n'en finit pas d'agacer sa pensée à sa lumière problématique, au lieu tout simplement d'y renoncer. Car les « juifs du retour » demeurent incontestablement endettés vis-à-vis du patrimoine philosophique de la modernité. Les prophètes de Chestov et de Fondane ne sont pas seulement les prophètes bibliques, ils se sont aussi appelés Lupasco ou Bachelard. Contemplant la tradition, Chestov, Fondane et Lévy font le pari qu'il y a, comme disait Lévinas, de l'or dans cette poussière, mais leur rétine, elle, demeure la rétine d'un Moderne. Quand bien même en appellent-ils comme Lévy à un retour orthodoxe à la tradition, c'est toujours arc-boutés à l'héritage des Lumières qu'ils instruisent le procès ; fidèles, jusque dans leurs blâmes et presque malgré eux, au projet moderne d'un usage critique de la raison. Comme si le retour continuait à répondre aux prestiges de l'histoire et de la philosophie occidentale quand bien même il s'en déclare affranchi, comme s'il continuait à se jouer dans l'espace de la pensée moderne.

Pour autant, les deux visions, celle de Chestov et Fondane dans l'entre-deuxguerres et celle de Benny Lévy après la Shoah demeurent fondamentalement dissemblables. S'agit-il en effet de sonder une pensée non moderne pour mener une critique constructive de la modernité qui profite aux contemporains - on plonge aux sources mais pour mieux refaire surface, confronter les trouvailles et partager le butin - ou s'agit-il de voir dans cette pensée non moderne une contrée exotique n'appartenant qu'aux valeureux explorateurs choisissant d'y élire domicile? Bref, quelle place ces critiques de la modernité laissent-elles à l'universel, à la dynamique d'un jeu en commun ?

\section{Quel universel ?}

Force est de constater que les tragédies du $\mathrm{XX}^{\mathrm{e}}$ siècle ont emporté avec elles l'ancienne confiance dans l'universalisme moderne et la raison impersonnelle qui le fondait. Une certaine idée de l'universel a été démasquée, celle qui consistait

15. L'expression fit florès parmi les journalistes pour évoquer le trajet de Benny Lévy. Un raccourci auquel Benny Lévy, agacé, opposait une lecture plus complexe : "Cette formule tant reprise à mon sujet, "De Mao à Moïse", est un lapsus profondément significatif. Je ne dis jamais de "Mao à Moïse", je dis de "Moïse à Mao" parce que la partie la plus importante, la plus intéressante de cet itinéraire, c'est du Caire à Paris. [...] Je suis passé des Moïse [Moïse le prophète et Maïmonide] à Paris, pour déchoir à Mao, et c'est cela qui est important » in Cahiers d'études lévinassiennes, Hors-série, 2005, p. 9. 
à conquérir l'universalité par la voie de l'abstraction plutôt que par celle de la singularité humaine. Le fameux mot d'ordre kantien « d'euthanasie du judaïsme ", appelé dans ce schéma à s'effacer pour laisser place à une pure religion morale, semble plus que jamais obsolète. Le siècle des "retours » en a sonné le glas. Mais ce scepticisme à l'endroit de l'Universel n'a pas seulement pesé sur la philosophie, il a aussi reconfiguré le lien même des intellectuels juifs à l'altérité et aux modèles politiques qui avaient tenté de l'organiser.

"Amour distant » à l'endroit des Nations, c'est en ces termes que Gershom Scholem repensait, au lendemain du génocide, l'ancien désir de symbiose. Le franco-judaïsme n'a pas davantage échappé à ce séisme. Chez Benny Lévy, et de manière extrêmement vigoureuse, la césure est interprétée comme une mise en congé de l'histoire universelle : défaite des promesses du franco-judaïsme, et d'une manière plus générale, de toutes les entreprises de conciliation du particularisme juif avec l'universalisme des Gentils. Alors que Léon Chestov et Benjamin Fondane gagnent Paris dans les années 1920 comme on reprend vie en une terre promise, - souvenons-nous du jeune Fondane tapissant les murs de sa chambre roumaine des numéros du Mercure de France, ou de la volonté qui fut la sienne de "faire souche » dans la langue française - Benny Lévy rompt avec cet effort : « le trait d'union juif-français, je ne connais pas, affirme-t-il, - quel bonheur! Je reste persuadé que la figure de l'Israélite français, historialement agonise ». La Galout, dès lors, n'est plus qu'une parenthèse malheureuse. Alors que Chestov et Fondane cherchaient, dans leur retour au questionnement religieux à ré-enraciner la légitimité de la tradition juive dans l'espace européen, alors qu'il y avait chez eux une manière de relire les grands auteurs comme un palimpseste, comme s'il s'agissait de retrouver les traces de l'héritage biblique derrière les grands noms de la pensée européenne, Benny Lévy "déracine " l'histoire juive du paysage européen et réduit drastiquement le champ de l'investigation : puissance rassasiante du Livre ; en un Livre, tous les livres nous promet-il. Là où les premiers entendaient souffler l'Esprit biblique jusque chez les penseurs les plus réfractaires à la Bible, Lévy devine le Veau d'or, pressent l'édulcoration philosophique du judaïsme, s'inquiète d'une culture faisant écran à la pure transcendance. Chez Chestov et Fondane, retour multipolaire, fidélité polygame; retour dont on retrouve la trace du côté d'Emmanuel Lévinas, opiniâtre à maintenir la pluralité des mondes. Benny Lévy, lui, préfère trancher pour s'affranchir : fin des conciliations inconfortables, fin des nuances difficiles et des tergiversations éprouvantes. "Seule la pierre de Jérusalem m'apaise » écrit l'éternel apatride; si la filiation se voit ranimée, c'est désormais sous le signe de l'unique et de l'immobile.

Chestov, Fondane et Lévy, autant de périples et d'intuitions connexes à bien des égards. Même manière dans leurs œuvres d'opposer Athènes et Jérusalem, même condamnation de la " trahison " de Philon ; même formulation d'une pensée de l'existence appelant à des vérités de chair... Avec eux, en effet, se jouent toutes les combinaisons d'un lien tranché et mille fois réinventé. Le retour n'est jamais 
qu'une ligne brisée, grosse des réécritures qui la remettent perpétuellement en jeu, bien loin des schémas linéaires que laissait supposer l'idée de "retour ». Pour autant, en réduisant l'aventure diasporique toute entière à l'aventure d'une corruption, Beny Lévy met à mal l'air de famille. Si sa critique n'aboutit pas au rejet de la démocratie libérale, si son retour ne liquide pas tous les acquis des Lumières modernes, si son œuvre peut même jouer, sans doute, comme une utile mise en garde contre toutes les formes d'affadissement de la tradition qui réduisent sa profondeur historique aux seuls slogans des droits de l'homme, il y a pourtant là un véritable point de basculement : N'y a-il pas en effet le risque d'une désertion du champ des responsabilités dans l'histoire ? Quelle place ce Retour laisse-t-il, et à la plasticité historique de la tradition, et à la respiration du monde ?

« Judaïsme interminable » (Yerushalmi), modernité interminable, telle est au fond la leçon double de ces retours : la modernité est aussi indéracinable que l'est la tradition. Pour le meilleur et pour le pire, elle s'impose, d'ores et déjà, comme notre héritage, et appelle, elle aussi, une fidélité pour en relayer les promesses. En tant que "modernes " la question demeure alors pour nous la suivante : quel enseignement la modernité peut-elle puiser dans ces « retours »? Il est indéniable que ces ré-émergences de certains motifs de la tradition religieuse, quand ils ne sont pas réinvestis dans une vision du monde totalisante comme celui de l'univers pré-moderne de la religion; quand ils acceptent de s'inscrire comme un possible parmi d'autres - c'est-à-dire quand ils acceptent de s'appliquer à eux-mêmes l'impératif de " limite » qu'ils souhaitent si ardemment voir intégré par la modernité - ; quand ils ne répudient pas l'universalisme moderne mais s'attachent simplement à le repenser, ont une certaine pertinence sociale et politique. Alors que l'universalisme kantien fondait sa cause sur l'autonomie morale du sujet, ces retours posent tous la question d'une légitimité de certaines formes d'hétéronomie dans notre modernité contre l'illusion d'une auto-fondation du sujet. Quand ils ne cèdent pas à la fureur isolationniste, mais tentent de faire dialoguer l'universel de la tradition juive et les grands universaux modernes, quand ils se maintiennent sur le vif tranchant de ce conflit, on peut penser qu'ils offrent alors à notre modernité, bien au-delà du lieu auquel ils s'attachent, une ressource intellectuelle précieuse. Ressource pour questionner le naufrage de la raison du siècle des Lumières à l'épreuve de la barbarie, ressource pour dénoncer une « certaine vision politique du monde » laissant libre cours à la « circulation louche de l'Absolu » du côté des religions séculières, ressource pour résister à la débâcle d'une culture évidée de sa dimension transcendante au profit d'une raison utilitaire ; ressource, enfin, pour renouer avec l'idée même de filiation. Reste que l'entreprise demeure périlleuse. Moderne par son malaise et les crises dont elle est issue - et dont elle garde la marche saccadée - la " pensée du retour » lutte ici avec l'ange de sa propre histoire.

Julia DAVID

Julyadavyd@aol.com 
Badet Muriel, 2002, «Saint Paul : chuter pour se convertir » in Genet-Delacroix Marie-

Claude, Gugelot Frédéric, Desbussons Frédérique (éds.), Les conversions comme formes et figures de la métamorphose. Mutations et transferts culturels, Paris, L'Harmattan.

BARANOFF-CHestov Nathalie, 1991, Vie de Léon Chestov. Tome I, L'homme du souterrain (1866-1929), Paris, Éditions de la Différence.

Benjamin Walter, 1979, Correspondance, (Trad. G. Petitdemange), Paris, Aubier.

Biale David, 2001, Gershom Scholem. Cabbale et contre-histoire, Nîmes, Éditions de l'Éclat. Bouretz Pierre, 2003, Témoins du futur. Philosophie et messianisme, Paris, Gallimard. Buber Martin, 1986, « Le mythe dans le judaïsme » in Judaïsme, (trad. Marie-José Jolivet), Paris, Verdier.

Fondane Benjamin, 1919, «Cuvinte despre un prieten » Lumea evree, 19.

-, 1999, "Signification de Dada" in Carassou Michel, Releanou Petre, Fondane et

l'avant-garde, Paris, Paris-Méditerranée, Fondation culturelle roumaine.

-, 2001, Images et livres de France, Paris, Éditions Paris-Méditerranée.

-, 1996, Le Mal des fantômes, Paris, Paris-Méditerranée/L’Éther vague.

Gross Benjamin, 1998, Les Lumières du retour, "Orot haTéshuva » du Rav Kook, Paris, Albin Michel.

Hervieu-LéGer Danièle, 1999, Le Pèlerin et le converti, Paris, Flammarion.

HöHN Gerhard, 1994, Heinrich Heine. Un intellectuel moderne, Paris, PUF.

IANCu Carol, 1978, Les Juifs en Roumanie. 1866-1919. De l'exclusion à l'émancipation,

Aix-en-Povence, Éditions de l'université de Provence.

Jutrin Monique, 1989, Benjamin Fondane ou le peuple d'Ulysse, Paris, Librairie A.-G. Nizet.

KafKa Franz, 1988, Lettres à Milena, Paris, Gallimard.

Lévy Benny, Alain Finkielkraut, 2006, Le Livre et les livres. Entretiens sur la laïcité, Paris, Verdier.

LÉvy Benny, 2003, Être juif, Paris, Verdier.

-, 2005a, La Cérémonie de la naissance, Paris, Verdier.

-, 2004, La Confusion des temps, Paris, Verdier.

-, 2002, Le Meurtre du pasteur. Critique de la vision politique du monde, Paris, Verdier.

-, 2005b, "Itinéraire » Cahiers d'études lévinassiennes, hors-série.

Mattenklott Gert, 1991, "Nietzsche dans les revues culturelles juives de langue allemande de 1900 à 1938 » in Bourel Dominique, Le Rider Jacques, De Sils Maria à Jérusalem.

Nietzsche et le judaïsme. Les intellectuels juifs et Nietzsche, Paris, Éditions du Cerf. Moses Stéphane, 1992, L'Ange de l'histoire. Rosenzweig, Benjamin, Scholem, Paris, Seuil. Rosenzweig Franz, 1935, Briefe, Berlin, Schoken.

Scholem Gershom, 1999, Aux origines religieuses du judaïsme lä̈c. De la mystique aux Lumières, Paris, Calmann-Lévy.

Steiner George, 2003, Les Logocrates, Paris, Éditions de l'Herne. 


\section{Résumé}

L'expérience de redéconverte du judaïsme telle qu'elle s'impose dans l'épreuve de la modernité à partir de la fin du XVIII ${ }^{e}$ siècle s'affirme paradoxale: opération de réinvestissement identitaire et culturel au lendemain des grandes fissurations de l'Émancipation, elle ne cesse pour autant d'illustrer le vertige d'une transmission problématique et d'un lien altéré; derviches tourneurs du rachat et de la reprise, ces phénomènes de ré-adhésion ou de "retour » à la tradition juive ne cessent d'inquiéter l'héritage. Des exigences particulières dictées par un statut minoritaire qui contraint les Juifs à résister aux séductions de l'assimilation, aux ébranlements du siècle qui ont si souvent conduit le judaïsme au bord du gouffre, ces cheminements à rebours apparaissent singulièrement malaisés.

C'est une double impossibilité - impossibilité de se dérober à l'histoire juive et impossibilité de la restaurer - qui conduit nombre d'intellectuels juifs sur le chemin de la critique de la modernité : la réponse ne sera pas seulement religieuse ; la "pensée du retour " se fait aussi critique sociale, indissociable d'une réflexion plus vaste sur les impasses du progressisme historique. Dans la "pensée du retour" coexistent en effet deux mouvements distincts et complémentaires: un élan de re-légitimation de la tradition juive, mais également une critique des promesses de l'Aufklärung et des philosophies du Progrès.

Mots-clés : pensée du retour, tradition juive, critique sociale, crise de la modernité, intellectuels juifs.

\section{Abstract}

The rediscovery of Judaism such as it was experienced in the face of modernity at the end of the Eighteenth Century turned out to be paradoxical: following the Emancipation and the cracks it had caused, it constituted an act of reinvestment as far as identity and culture were concerned, and yet, ever since, it has revealed the uncertainties of a problematic transmission and an altered bond. The phenomena of return and re-adhesion to Jewish tradition, which can be seen as the whirling dervishes of repurchase and revival and as the will to perform the inaugural once again, are phenomena which constantly nag at memory and disturb legacy. From the specific demand, dictated by their minority status, that the Jews resist the temptation of assimilation, to the deeply unsettling events of the century, which so often pushed the Jews to the brink of the abyss, there are scores of reasons to perceive those backward-going processes as particularly difficult.

We are faced with a double impossibility: of ignoring Jewish history on the one hand, and of restoring it on the other, and this very double impossibility led so many Jewish intellectuals to criticise modernity. Indeed, their response was not to be a solely religious one: the "philosophy of return" was also a form of social criticism which couldn't do without tackling a broader issue, namely the dead-ends reached by historical progressivism. For, in the philosophy of return, two trends coexist, both separate and complementary and always interrelated: an attempt at re-legitimizing Jewish traditions, and a certain wariness of the promises of the Aufklärung and of the philosophies of Progress. (Trad. Marion Roman)

Key words: philosophy of return, Jewish tradition, social criticism, crisis of modernity, Jewish thinkers. 


\section{Resumen}

La experiencia del redescubrimiento del judaísmo, tal como se impone ante el desafío de la modernidad a partir de fines del siglo XVIII, resulta paradójica: operando un reforzamiento identitario y cultural posterior a las grandes fisuras de la Emancipación, no deja, sin embargo, de ilustrar el vértigo de una transmisión problemática y de un vínculo alterado; movimientos giratorios de reapropiación y de reanudación, estos fenómenos de re-adhesión o de un "retorno" a la tradición judia no hacen más que inquietar a la tradition. Exigencias particulares dictadas por un estatuto minoritario que obliga a los judios a resistir a las seducciones de la asimilación, a los estremecimientos del siglo que con frecuencia condujeron al judaísmo al borde del abismo, estas caminatas a tientas parecen ser especialmente incómodas.

Se trata de una doble imposibilidad - de escabullirse de la historia judía e de restaurarlaque conduce a un número importante de intelectuales judios por el camino de la crítica de la modernidad: la respuesta, en efecto, no será sólo religiosa; el "pensamiento del retorno" se transforma también en crítica social, indisociable de una reflexión más vasta sobre las dificultades del progresismo histórico. De hecho, en el "pensamiento del retorno" coexisten dos movimientos distintos y complementarios : un impulso de relegitimación de la tradición judia, ya sea manifestándose así como una crítica de las promesas de l'Aufklärung y de las filosofías del Progreso. (Trad. Consuelo Biskupovic, Ens/Ehess)

Palabras clave : el pensamiento del retorno, tradición judía, critica social, crisis de la modernidad, intelectuales judios. 
